\title{
Rising Trend in Thyroid Cancer - A Truth Or Myth? - A Prospective Study in a Tertiary Care Hospital in Southern India
}

\author{
Dr.K.Poongkodi ${ }^{1}$ M.S., M.Ch, Prof.Dr.N.Tamilselvan ${ }^{2}$ M.S., \\ Prof.Dr.Thenmozhi ${ }^{3}$ M.D., \\ ${ }^{1}$ Assistant Professor, Department of General Surgery, Government Mohankumaramangalam Medical College, \\ Tamilnadu Dr MGR Medical University, India \\ ${ }^{2}$ Associate Professor, Department of General Surgery, Government Mohankumaramangalam Medical College, \\ Tamilnadu Dr MGR Medical University, India \\ ${ }^{3}$ Professor, Department of Pathology, Government Mohankumaramangalam Medical College, Tamilnadu Dr \\ MGR Medical University, India
}

\begin{abstract}
:
Background: The incidence of thyroid cancer has been increasing several-fold over the decades while other head and neck cancers are decreasing. Whether the increasing incidence of thyroid cancer is a true disease occurrence or due to detection by increased diagnostic scrutiny remains a subject of debate. Hence, we prospectively evaluated the trends in the thyroid cancer incidence, their clinicopathological features and histology.

Methods And Materials: All Patients admitted for thyroid surgery in the year 2014, 2015 and 2016 were included in the group A (n=105), B $(n=112)$ and $C(n=45)$ respectively. Age, sex, thyroid profile, cytology, ultrasound, tumor stage and extent of surgery were studied. Final diagnosis was confirmed with histopathological examination. Recurrence and mortality were noted in the follow up of 19.6+/-8months. Statistical analysis was performed with SPSS. P<0.05 was considered significant.

Results: Out of 262 patients (male: female=17:245) who underwent thyroid surgery, 42 patients had cancer (Incidence $=16.07 \%$ ). The incidence of thyroid carcinoma for the year 2014, 2015 and 2016 were $13.4 \%, 16.9 \%$ and $19.5 \%$ respectively, $p<0.05$. Papillary thyroid carcinoma, the most common type constituted $92.8 \%$ of all thyroid cancer cases. Significant differences in age and extent of surgery was observed ( $p=0.03$ and $p 0.015$ respectively). Increased incidence was observed across all age, men and women and tumor size $>4 \mathrm{~cm}$.

Conclusion: The Rising trend in the thyroid cancer is due to true disease occurrence, attributed by the increasing incidence of Papillary thyroid carcinoma across all ages, gender and tumor stages. Environmental influences and molecular pathways need further investigation.
\end{abstract}

Keywords: Papillary thyroid carcinoma, Thyroid cancer incidence, Total thyroidectomy, secular trend

\section{Introduction}

Thyroid cancer is relatively uncommon, accounting only $1 \%$ of all malignancy [1]. However, thyroid cancer is the most common endocrine malignancy worldwide and its incidence has been increasing many-fold over the decades. The incidence of thyroid cancers has increased 2.4 fold from 3.6 per 100000 persons in 1973 in the United States to 8.7 per 100000 in 2002 [2]. The rising trend of thyroid cancer is due to the increasing incidence of papillary thyroid carcinoma (PTC), which showed a 2.9 fold increase from 2.7 to 7.7 per 100000 persons whereas the incidence rates of follicular, anaplastic, and medullary cancers were stable. Nearly half of the new cancers measured $1 \mathrm{~cm}$ or smaller, and $87 \%$ were $2 \mathrm{~cm}$ or smaller. This trend has also been noted in many other countries across Europe, Asia, Oceania, and South America [3]. The only countries to notice a decline in thyroid cancer rates in Europe were Sweden, Norway, and Spain. The incidence of PTC is also increasing among children at $1.1 \%$ per year in US [4] and over 3\% across Europe [5]. Many investigators believe that the rising trend in thyroid cancer is not true disease occurrence but apparent due to the detection by increased diagnostic scrutiny [6,7]. The advent of high frequency ultrasound and fine needle aspiration has led to the increased detection of cancer from thyroid incidentaloma, exposing the subclinical reservoir. On the other hand, some investigators [8-11] suggest that the rising trend is true and observed an increasing rate of cancer incidence across all tumor stages, even tumors $>\mathrm{cm}$. They reported increased cancer incidence rates across all age groups among both men and women.

Whether the increasing incidence of thyroid cancer is a true disease occurrence or due to detection by increased diagnostic scrutiny still remains controversial. Hence, we designed this prospective study to evaluate the trends in the thyroid cancer incidence, their clinicopathological features, histology and size distribution in 
the Tertiary Care hospital, which serves the hilly terrain of the neighbouring seven districts, wherein the cancer incidence rates remains unraveled.

\section{Materials And Methods}

All subjects admitted for thyroid surgery consecutively in the department of General Surgery, Government Mohankuramangalam Medical College hospital from January 2014 till June 2016 was included in the study. Subjects admitted in the year 2014 were categorized as Group A, while those admitted in the year 2015 and 2016 were included in group B and group C respectively. Informed consent obtained. Age, gender, thyroid profile, sonographic features, fine needle cytology (FNAC) of the thyroid nodule was studied. In case of retrosternal extension or suspected malignancy, computed tomography of the neck was performed. Indications for surgery were large volume goitres, presence of compressive symptoms, retrosternal extension, suspicion of malignancy, cosmesis, Graves' disease with ophthalmopathy, cardiotoxicity or patient's preference for surgery and other logistic reasons. Patients with benign thyroid cysts, pregnant women with physiological goitre who were not surgical candidates were excluded from the study. Hemithyroidectomy, subtotal thyroidectomy, near total and Total thyroidectomy (TT) were the procedures performed tailored to individual needs. However, TT is the procedure of choice for Graves' disease by protocol. TT or TT with therapeutic central compartment and lateral compartment neck dissection was performed for thyroid cancers. Completion thyroidectomy is carried out if hemithyroidectomy specimens of follicular neoplasm harbours cancer foci and in cases of recurrence along with appropriate lymphadenectomy. Thyroid cancer histology noted. Tumor size distribution was defined as less than $1 \mathrm{~cm}$ in greatest dimension, $1 \mathrm{~cm}$ to $<2 \mathrm{~cm}, 2 \mathrm{~cm}$ to $<4 \mathrm{~cm}$ and $>=4 \mathrm{~cm}$. Tumor staging was done as localized if confined to thyroid, invasive if infiltration of surrounding structures with lymph-node spread and distant in the presence of distant metastasis based on SEER (Surveillance, Epidemiology, End Results) database. Patients received supplementary, replacement or suppressive dose of Thyroxine sodium based on extensiveness of thyroidectomy and their histology. A few cases of differentiated thyroid cancers (DTC) received 131-I radioactive iodine remnant ablation or therapy for distant metastases. These subjects are monitored till date with TSH, serum Thyroglobulin and imaging on suspicion of recurrence.

\section{Statistical Analysis}

Statistical analysis was performed with SPSS software version 20. Student's T test, analysis of variance and regression was used where appropriate. $\mathrm{P}$ value of less than 0.05 was considered significant.

\section{Results}

During the study period, 262 subjects (male: female=17:245) underwent thyroid surgery. Out of these 262 subjects, thyroid cancer was detected in 42 patients on final histopathological examination (Cancer Incidence $=16.07 \%$ ). In Group A, 14 of 105 patients (M: F=5:100) had cancer. In group B, 19 of 112 patients $(\mathrm{M}: \mathrm{F}=6: 106)$ had cancer. Among Group $\mathrm{C}, 9$ of 45 patients $(\mathrm{M}: \mathrm{F}=6: 39)$ had cancer. Thus, the incidence of thyroid carcinoma for the year 2014, 2015 and 2016 were 13.4\%, 16.9\% and $19.5 \%$ respectively, p<0.05 and increased over the years. The clinicopathological features of these 42 cancer patients are given in the Table 1 . Papillary thyroid carcinoma (PTC) was the most common histological type and constituted $92.8 \%$ of all thyroid cancer cases. In nearly one-third of these cases, Hashimoto's thyroiditis was the coexistent pathology followed by adenomatous hyperplasia. One case of anaplastic thyroid carcinoma was diagnosed in a 70 year old male on core needle biopsy and referred to the department of radiation oncology for favour of radiotherapy. Two cases of follicular thyroid cancer were identified. Differences in age and extent of surgery ( $\mathrm{p}=0.03$ and $\mathrm{p} 0.015$ respectively) was statistically significant between the groups. (i.e.) preference for more radical procedure from hemithyroidectomy and subtotal thyroidectomy to TT for even benign thyroid diseases was observed towards 2016. Similarly TT and TT with neck dissection increased over the years for DTCs. Increased incidence of thyroid cancer was observed across all age groups in both gender, namely men and women.

\section{Discussion}

In our study, we observed an increase in the incidence of thyroid cancers over the years from 2014 till date. PTC was the most common histological type and represented over $92.8 \%$ of all thyroid cancer cases. Thus, the increase in the incidence of thyroid cancer is virtually entirely attributed to the increasing incidence of papillary thyroid cancer in our study. We demonstrated an increased cancer rates across all age groups ranging from 7 yrs to 70 yrs of age. Perhaps, a few cases of PTC have been reported in children below 10 yrs of age in India. To the best of our knowledge, this is probably the second case of PTC in a 7 year old girl to be reported from Southern India. However, we observed an age shift with peak occurrence around 35 years of age. Our study also showed an increase in thyroid cancers of all sizes, even $>4 \mathrm{~cm}$. This is in contrast to the most of the published evidence that rising trend is attributed to the increased incidence of PTC less than $2 \mathrm{~cm}$. This is probably due to the fact that our hospital is the tertiary care referral centre for the neighbouring seven districts in 
the hilly terrain zone. Most of the patients presented with symptomatic neck mass and referred for expert management. This contributes to the referral bias and provides explanation for the higher stage tumors observed in our study. In support of our observation, Chen et al. reported increased incidence of DTC of all sizes among both men and women [8].

The shift to more radical surgical techniques such as total thyroidectomy for many of the benign thyroid diseases has increased the detection of incidental tumors. In addition, alteration in pathological techniques such as 2 to $3 \mathrm{~mm}$ sectioning of thyroidectomy specimens has increased the detection of small foci of tumors previously missed. Some investigators attributed the increasing incidence to exposure to increasing radiation and endocrine disrupting chemicals such as polybrominated diphenyls. However, supportive evidence of increase in such risk factors is lacking. Given the stable mortality in the face of increasing cancer incidence, some investigators concluded increased case detection of smaller, lower stage PTC by diagnostic scrutiny hence excellent prognosis [2]. On the other hand, better understanding of the natural history of thyroid cancer, multidisciplinary approach with improved surgical techniques, availability of Thyroglobulin assays, recombinant Thyroglobulin, PET/CT scans and radioiodine therapy, could also be a possibility for the stable cancer mortality rates despite the increasing cancer incidence.

Interestingly, in our study, in nearly one-third of our cases Hashimoto's thyroiditis was the coexistent pathology. Larson et al reported that patients with Hashimoto thyroiditis (a condition in which PI3K/Akt expression increased) were 3 times more likely to develop thyroid cancer [12]. The causal relationship between Hashimoto's thyroiditis and PTC and their molecular pathways needs further investigation.

\section{Tables}

Table.1. Clinicapathologic features of cancer patients in each group

\begin{tabular}{|c|c|c|c|c|c|}
\hline PARAMETER & $\mathbf{A}$ & $\mathbf{B}$ & $\mathbf{C}$ & & $\mathrm{P}$ value \\
\hline No. of cancer patients & 14 & 19 & 9 & 42 & \\
\hline AGE (mean+/-SD) years & $37.2+/ 17.1$ & $41.3+/-12.1$ & $29.8+/-14.4$ & & 0.035 \\
\hline Age (range) years & 7 to 65 & 23 to 70 & 14 to 45 & & \\
\hline$<40 \mathrm{Y}$ & 8 & 9 & 7 & 24 & \\
\hline \multirow{2}{*}{$>40 \mathrm{Y}$} & 6 & 10 & 2 & 18 & \\
\hline & 14 & 19 & 9 & 42 & \\
\hline \multicolumn{6}{|l|}{ GENDER } \\
\hline Female & 10 & 16 & 7 & 33 & \multirow[t]{3}{*}{0.66} \\
\hline \multirow[t]{2}{*}{ Male } & 4 & 3 & 2 & 9 & \\
\hline & 14 & 19 & 9 & 42 & \\
\hline \multicolumn{6}{|l|}{ PROCEDURE } \\
\hline Hemithyroidectomy & 3 & 3 & 0 & 6 & \multirow[t]{5}{*}{0.015} \\
\hline Subtotal thyroidectomy & 3 & 5 & 0 & 8 & \\
\hline Total thyroidectomy & 5 & 10 & 6 & 21 & \\
\hline TT neck dissection & 1 & 0 & 2 & 3 & \\
\hline \multirow[t]{2}{*}{ Completion thyroidectomy } & 2 & 1 & 1 & 4 & \\
\hline & 14 & 19 & 9 & 42 & \\
\hline \multicolumn{6}{|l|}{ TUMOR } \\
\hline$<1 \mathrm{~cm}$ & 3 & 3 & 2 & 8 & \multirow[t]{4}{*}{0.34} \\
\hline 1 to $1.9 \mathrm{~cm}$ & 4 & 5 & 1 & 10 & \\
\hline 2 to $3.9 \mathrm{~cm}$ & 5 & 6 & 3 & 14 & \\
\hline \multirow[t]{2}{*}{4 or $>4 \mathrm{~cm}$} & 2 & 5 & 3 & 10 & \\
\hline & 14 & 19 & 9 & 42 & \\
\hline \multicolumn{6}{|l|}{ STAGE(SEER) } \\
\hline LOCAL & 8 & 11 & 3 & 22 & \multirow[t]{3}{*}{0.66} \\
\hline INVASIVE & 5 & 6 & 6 & 17 & \\
\hline \multirow[t]{2}{*}{ DISTANT } & 1 & 2 & 0 & 3 & \\
\hline & 14 & 19 & 9 & 42 & \\
\hline
\end{tabular}

\section{Conclusion}

Thus, we conclude that the increasing incidence of thyroid cancer is due to true disease occurrence, attributed by the increasing incidence of Papillary thyroid carcinoma across all age, gender and tumor stage. This study has a few limitations. The study was performed for a shorter duration and needs long-term evaluation over decades to assess the secular trend of cancer incidence rates. Environmental influences and molecular pathways need further investigation. Furthermore, the co-existence of Hashimoto's thyroiditis and PTC needs prospective studies to evaluate their causal association.

\section{Conflict Of Interest}

The authors declared no conflict of interest. 


\section{References}

[1]. J.A. Sipos ELM. Thyroid cancer epidemiology and prognostic variables. Clin Oncol (R Coll Radiol), 22, $2010,395-404$.

[2]. Davies L, Welch HG. Increasing incidence of thyroid cancer in the United States, 1973-2002. JAMA, 295(18), 2006, 2164-7.

[3]. Kilfoy BA, Zheng T, Holford TR, Han X, Ward MH, Sjodin A, et al. International patterns and trends in thyroid cancer incidence, 1973-2002. Cancer Causes Control. 2009 Jul;20(5):525-31.

[4]. A.R. Hogan YZ, E.A. Perez 2009. Pediatric thyroid carcinoma: incidence and outcomes in 1753 patients. J Surg Res, 156, 2009, 167-72.

[5]. E. Steliarova-Foucher CAS, E. Pukkala. Thyroid cancer incidence and survival among European children and adolescents (19781997): report from the Automated Childhood Cancer Information System project. Eur J Cancer, 42, 2006, $2150-69$.

[6]. Hall SF, Walker H, Siemens R, Schneeberg A. Increasing detection and increasing incidence in thyroid cancer. World J Surg, 33(12), 2009, 2567-71

[7]. Griniatsos J, Tsigris C, Kanakis M, Kaltsas G, Michail O, Dimitriou N, et al. Increased incidence of papillary thyroid cancer detection among thyroidectomies in Greece between 1991 and 2006. Anticancer Res, 29(12), 2009, 5163-9.

[8]. Chen AY, Jemal A, Ward EM. Increasing incidence of differentiated thyroid cancer in the United States, 1988-2005. Cancer, 115(16), 2009, 3801-7.

[9]. Bernal M, Gomez GJ, Gomez FJ. [Increase of thyroid cancer incidence]. Med Clin (Barc), 133(11), 2009, 442-3.

[10]. Leenhardt L, Grosclaude P, Cherie-Challine L. Increased incidence of thyroid carcinoma in France: a true epidemic or thyroid nodule management effects? Report from the French Thyroid Cancer Committee. Thyroid, 14(12), 2004, 1056-60.

[11]. Leenhardt L, Bernier MO, Boin-Pineau MH, Conte Devolx B, Marechaud R, Niccoli-Sire P, et al. Advances in diagnostic practices affect thyroid cancer incidence in France. Eur J Endocrinol, 150(2), 2004, 133-9.

[12]. Larson SD, Jackson LN, Riall TS, Uchida T, Thomas RP, Qiu S, et al. Increased incidence of well-differentiated thyroid cancer associated with Hashimoto thyroiditis and the role of the PI3k/Akt pathway. J Am Coll Surg, 204(5), 2007, 764-75. 